\title{
Thyroid carcinoma: molecular pathways and therapeutic targets
}

\author{
Yuri E Nikiforov \\ Department of Pathology and Laboratory Medicine, University of Pittsburgh Medical Center, Pittsburgh, \\ $P A, U S A$
}

\begin{abstract}
Thyroid cancer is the most common malignant tumor of the endocrine system. The most frequent type of thyroid malignancy is papillary carcinoma. These tumors frequently have genetic alterations leading to the activation of the mitogen-activated protein kinase (MAPK) signaling pathway. Most common mutations in papillary carcinomas are point mutations of the BRAF and RAS genes and RET/PTC rearrangement. These genetic alterations are found in $>70 \%$ of papillary carcinomas and they rarely overlap in the same tumor. Most frequent alterations in follicular carcinomas, the second most common type of thyroid malignancy, include RAS mutations and PAX8-PPARy rearrangement. RET point mutations are crucial for the development of medullary thyroid carcinomas. Many of these mutations, particularly those leading to the activation of the MAPK pathway, are being actively explored as therapeutic targets for thyroid cancer. A number of compounds have been studied and showed antitumor effects in preclinical studies and are being tested in ongoing clinical trials. Modern Pathology (2008) 21, S37-S43; doi:10.1038/mpath.2008.10
\end{abstract}

Keywords: thyroid cancer; mutations; MAPK; BRAF; RET; inhibitors

Thyroid cancer is the most common malignant tumor of the endocrine system and accounts for approximately $1 \%$ of all newly diagnosed cancer cases. ${ }^{1}$ The most frequent type of thyroid malignancy is papillary carcinoma, which constitutes $\sim 80 \%$ of all cases. Papillary carcinomas frequently have genetic alterations leading to the activation of the mitogen-activated protein kinase (MAPK) signaling pathway (Figure 1). Those include RET/ PTC rearrangement and point mutations of the $B R A F$ and $R A S$ genes. Mutations involving one of these genes are found in $>70 \%$ of papillary carcinomas and they rarely overlap in the same tumor $^{2-4}$ (Table 1). Frequent genetic alterations in follicular carcinomas, the second most common type of thyroid malignancy, include $R A S$ mutations and $P A X 8-P P A R \gamma$ rearrangement. RET point mutations are crucial for the development of medullary thyroid carcinomas. Many of these mutations, particularly those leading to the activation of the MAPK pathway, are being actively explored as therapeutic targets for thyroid cancer.

Correspondence: Dr YE Nikiforov, MD, PhD, Department of Pathology, University of Pittsburgh, A713 Scaife Hall, 3550 Terrace Street, Pittsburgh, PA 15261, USA.

E-mail: nikiforovye@upmc.edu

Received 6 December 2007; accepted 31 December 2007

\section{BRAF}

BRAF serine-threonine kinase belongs to the family of RAF proteins, which are intracellular effectors of the MAPK signaling cascade. Upon activation triggered by RAS binding and protein recruitment to the cell membrane, these kinases phosphorylate and activate MEK, which in turn activates ERK and consequent effectors of the MAPK cascade.

Point mutations of the BRAF gene are found in $\sim 45 \%$ of thyroid papillary carcinomas. ${ }^{2,5}$ Virtually all point mutations involve nucleotide 1799 and result in a valine-to-glutamate substitution at residue 600 (V600E) ${ }^{6,7}$ BRAF V600E mutation leads to constitutive activation of BRAF kinase and the mechanism of activations has been recently elucidated. In the dephosphorylated, wild-type BRAF protein, the hydrophobic interactions between the activation loop and the ATP binding site maintain the protein in an inactive conformation. The V600E substitution disrupts these interactions and allows the formation of new interactions that keep the protein in a catalytically competent conformation, resulting in continuous phosphorylation of MEK. ${ }^{8}$

$B R A F$ mutations are highly prevalent in papillary carcinomas with classical histology and in the tall cell variant, but are rare in the follicular variant. ${ }^{6,9} \mathrm{In}$ many studies, the presence of BRAF mutation has been found to correlate with aggressive tumor characteristics such as extrathyroidal extension, advanced tumor stage at presentation, tumor recurrence, 


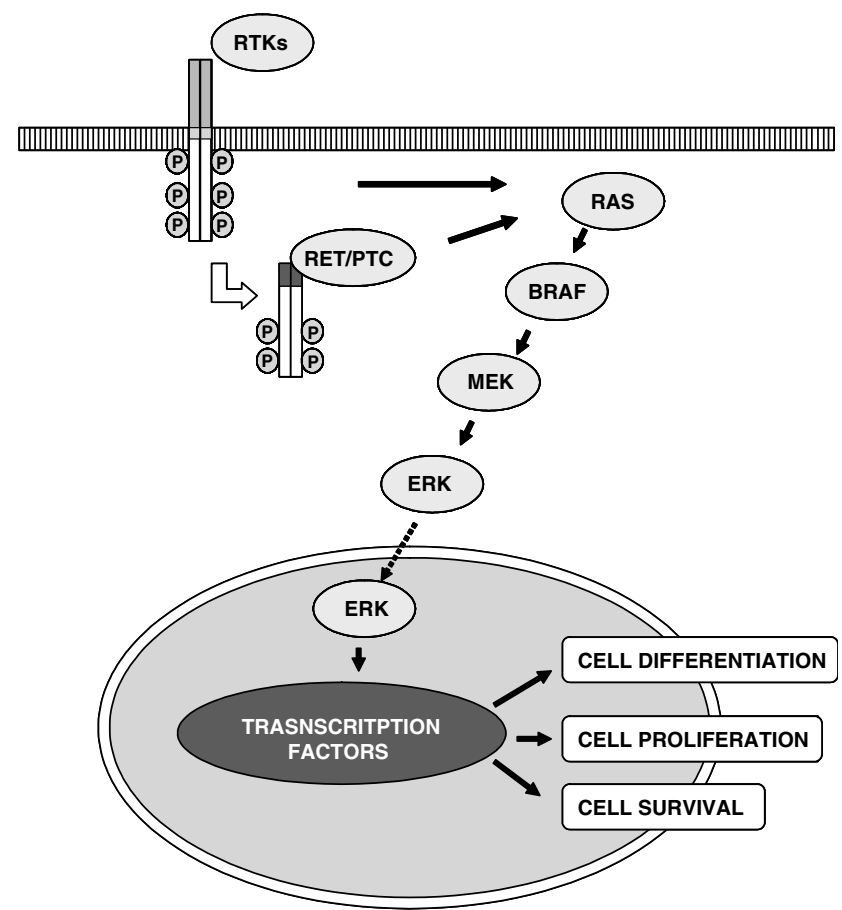

Figure 1 MAPK signaling pathway is physiologically activated by binding of growth factors to receptor tyrosine kinases (RTKs), such as RET and NTRK, resulting in receptor dimerization and activation via autophosphorylation of tyrosine residues in the intracellular domain. The activated receptor, through a series of adaptor proteins, leads to activation of RAS located at the inner face of the plasma membrane. The activated RAS binds to and recruits RAF proteins (mainly BRAF in thyroid follicular cells) to the plasma membrane. Activated BRAF phosphorylates and activate the MAPK/ERK kinase (MEK), which in turn phosphorylates and activates the extracellular signal-regulated kinase (ERK). Activated ERK translocates into the nucleus, where it regulates transcription of the genes involved in cell differentiation, proliferation, and survival. Alterations of this pathway in thyroid cancer can occur at different levels as a result of point mutation or rearrangement involving the RET, RAS, and BRAF genes.

and lymph node or distant metastases. ${ }^{10-12}$ Importantly, BRAF V600E has been found to be an independent predictor of tumor recurrence even in patients with stage I-II of the disease. ${ }^{12,13} B R A F$ mutations have also been associated with the decreased ability of tumors to trap radioiodine and treatment failure of the recurrent disease, which may be due to the dysregulation of function of the sodium iodide symporter (NIS) and other genes metabolizing iodide in thyroid follicular cells. ${ }^{12,14}$

Other and rare mechanisms of BRAF activation in papillary thyroid cancer include K601E point mutation, small in-frame insertions or deletions surrounding codon $600,,^{15-17}$ and AKAP9-BRAF rearrangement, which is more common in papillary carcinomas associated with radiation exposure. ${ }^{18}$

In addition to papillary carcinomas, $B R A F$ is found mutated in thyroid anaplastic and poorly differentiated carcinomas, typically in those tumors that also contain areas of well-differentiated papillary carcinoma. ${ }^{10,11,19}$ In these tumors, $B R A F$ mutation is detectable in both well-differentiated and poorly differentiated or anaplastic tumor areas, providing evidence that it occurs early in tumorigenesis.

\section{RETIPTC}

The RET proto-oncogene codes for a cell membrane receptor tyrosine kinase. In the thyroid gland, RET is highly expressed in parafollicular C-cells but not in follicular cells, where it can be activated by chromosomal rearrangement known as RET/PTC rearrangement. ${ }^{20,21}$ In RET/PTC, the $3^{\prime}$ portion of the RET gene is fused to the $5^{\prime}$ portion of various unrelated genes. At least 11 types of RET/PTC have been reported to date, all formed by the RET fusion to different partners. ${ }^{22,23}$ The two most common rearrangement types, RET/PTC1 and RET/PTC3, account for the vast majority of all rearrangements found in papillary carcinomas. RET/PTC1 is formed by fusion with the H4 (D10S170) gene, and RET/ PTC3 by fusion with the NCOA4 (ELE1) gene. ${ }^{21,24}$ The fusion leaves intact the TK domain of the RET receptor and enables the RET/PTC oncoprotein to bind SHC and activate the RAS-RAF-MAPK cascade. ${ }^{25}$

$R E T / P T C$ is tumorigenic in thyroid follicular cells, as it transforms thyroid cells in culture ${ }^{26}$ and gives rise to thyroid carcinomas in transgenic mice. ${ }^{27-29}$ Several studies suggest that the oncogenic effects of RET/PTC require signaling along the MAPK pathway and the presence of the functional BRAF kinase. ${ }^{25,30,31}$ Indeed, BRAF silencing in cultured thyroid cells reverses the RET/PTCinduced effects such as ERK phosphorylation, inhibition of thyroid-specific gene expression, and increased cell proliferation. ${ }^{30,31}$

$R E T / P T C$ is found on average in $\sim 20 \%$ of adult sporadic papillary carcinomas, although its prevalence is highly variable between different observations. ${ }^{22,23}$ In general, RET/PTC incidence is higher in tumors from patients with a history of radiation exposure and in pediatric populations. The distribution of RET/PTC rearrangement within each tumor may vary from involving almost all neoplastic cells (clonal RET/PTC) to being detected only in a small fraction of tumor cells (non-clonal RET/ PTC). ${ }^{32,33}$ The heterogeneity may be of a potential problem for the RET receptor-targeted therapy, since tumors with non-clonal RET/PTC frequently have other genetic alterations and may not respond to RET inhibitors in the same way as tumors harboring the clonal rearrangement.

Papillary carcinomas with RET/PTC rearrangements typically present at younger age and have a high rate of lymph node metastases, classic papillary histology, and possibly more favorable prognosis, particularly those harboring $R E T / P T C 1 .{ }^{9}$ In tumors arising after radiation exposure, RET/PTC1 
Table 1 Average prevalence of mutations in thyroid cancer

\begin{tabular}{lc}
\hline Tumor type & Prevalence (\%) \\
\hline Papillary carcinoma & \\
BRAF & 45 \\
RET/PTC & 20 \\
RAS & 10 \\
TRK & $<5$ \\
& \\
Follicular carcinoma & \\
RAS & 45 \\
PAX8-PPAR & 35 \\
PIK3CA & $<10$ \\
PTEN & $<10$ \\
& \\
Medullary carcinoma & \\
Familial forms of RET & $>95$ \\
Sporadic RET & 50 \\
& \\
Poorly differentiated carcinoma & \\
RAS & \\
$\beta$-Catenin (CTNNB1) & 35 \\
TP53 & 20 \\
BRAF & 20 \\
Anaplastic carcinoma & 15 \\
TP53 & \\
$\beta$-Catenin (CTNNB1) & \\
RAS & \\
BRAF & 70 \\
\hline
\end{tabular}

was found to be associated with classic papillary histology, whereas RET/PTC3 type was more common in the solid variants. ${ }^{34}$

\section{RAS}

The RAS genes (H-RAS, K-RAS, and N-RAS) encode highly related G-proteins that are located at the inner surface of the cell membrane and play a central role in the intracellular transduction of signals arising from cell membrane receptors tyrosine kinase and G-protein-coupled receptors. In its inactive state, RAS protein is bound to guanosine diphosphate (GDP). Upon activation, it releases GDP and binds guanosine triphosphate (GTP), activating the MAPK and other signaling pathway, such as PI3K/AKT. Normally, the activated RAS-GTP protein becomes quickly inactive due to its intrinsic guanosine triphosphatase (GTPase) activity and the action of cytoplasmic GTPase-activating proteins, which catalyze the conversion of the active GTP form to the inactive GDP-bound form. In many human neoplasms, point mutations occur in the discrete domains of the RAS gene, which result in either an increased affinity for GTP (mutations in codons 12 and 13) or inactivation of the autocatalytic GTPase function (mutations in codon 61). As a result, the mutant protein becomes permanently switched in the active position and constitutively activates its downstream signaling pathways.
Point mutations of RAS occur with variable frequency in all types of thyroid follicular cell-derived tumors. In papillary carcinomas, RAS mutations are relatively infrequent, as they occur in $\sim 10 \%$ of tumors. ${ }^{35,36}$ Papillary carcinomas with RAS mutations almost always have the follicular variant histology; this mutation also correlates with significantly less prominent nuclear features of papillary carcinoma, more frequent encapsulation, and low rate of lymph node metastases.9,37 Some studies have reported the association between $R A S$ mutations and more aggressive behavior of papillary carcinoma and with higher frequency of distant metastases. ${ }^{38}$ In follicular thyroid carcinomas, $R A S$ mutations are found in $40-50 \%$ of tumors ${ }^{39-41}$ and may also correlate with tumor dedifferentiation and less favorable prognosis. ${ }^{42,43} R A S$ mutations may predispose to tumor dedifferentiation, as they are found with high prevalence in anaplastic (undifferentiated) thyroid carcinomas. This may be due to the effect of mutant RAS to promote chromosomal instability, which has been documented in the in vitro setting. ${ }^{44,45}$ RAS mutations, however, are not specific for thyroid malignancy and also occur in benign follicular adenomas.

\section{PAX8-PPAR}

PAX8-PPAR $\gamma$ rearrangement results from the translocation $\mathrm{t}(2 ; 3)(\mathrm{q} 13 ; \mathrm{p} 25)$ that leads to the fusion between the PAX8 gene, which encodes a paired domain transcription factor, and the peroxisome proliferator-activated receptor $(P P A R \gamma)$ gene. $^{46}$ $P A X 8-P P A R \gamma$ occurs in $\sim 35 \%$ of conventional follicular carcinomas, and with lower prevalence in oncocytic (Hurthle cell) carcinomas. ${ }^{47-49}$ Tumors harboring $P A X 8-P P A R \gamma$ tend to present at a younger age, be smaller in size, and more frequently have vascular invasion. The rearrangement results in overexpression of the PPAR $\gamma$ protein that can be detected by immunohistochemistry. ${ }^{46,50}$

The mechanisms of cell transformation induced by PAX8-PPAR $\gamma$ are not fully understood. Some evidence has been presented for inhibition of normal PPAR $\gamma$ function via a dominant-negative effect of the PAX8-PPAR $\gamma$ protein on wild-type PPAR $\gamma .{ }^{46,51}$ Other studies have found the activation of known PPAR target genes in tumors harboring $P A X 8-P P A R \gamma$, arguing against the dominant-negative effect. ${ }^{52}$ Other possible mechanisms include deregulation of PAX8 function, known to be critical for thyroid cell differentiation, and activation of a set of genes related to neither wild-type PPAR $\gamma$ nor wildtype PAX8 pathways. ${ }^{52,53}$

$P A X 8-P P A R \gamma$ rearrangements and $R A S$ point mutations rarely overlap in the same tumor, suggesting that follicular carcinomas may develop via at least two distinct molecular pathway, initiated by either $P A X 8-P P A R \gamma$ or $R A S$ mutation. ${ }^{48}$ 


\section{RET point mutations}

Alteration of the RET proto-oncogene plays a causal role in the familial forms of medullary thyroid carcinoma and has also been found in sporadic forms of the disease. In medullary carcinomas, RET is activated by point mutation, in contrast to its activation by chromosomal rearrangement in papillary thyroid carcinomas. Germline mutations in the discrete functional regions of $R E T$ are found in almost all patients with familial forms of medullary carcinoma. In MEN 2A and familial medullary carcinoma, mutations are typically located in one of five cysteine codons within the cysteine-rich extracellular domain. ${ }^{54}$ Almost $90 \%$ of MEN 2A mutations affect codon 634, whereas in familial medullary carcinoma they are more evenly distributed along the cysteine-rich region. ${ }^{55}$ These mutations result in unpairing of cysteine residues in the extracellular domain, leading to the formation of disulfide bonds between two mutated receptor molecules and ligand-independent dimerization and constitutive activation of RET kinase. ${ }^{56}$ In MEN 2B, the majority of germline mutations occur in codon 918 in the intracellular tyrosine kinase domain of RET. This mutation is believed to alter the substrate specificity of RET kinase, resulting in phosphorylation of unusual intracellular proteins. ${ }^{56}$

In sporadic medullary carcinomas, somatic mutations of $R E T$ are found in $20-80 \%$ of cases. ${ }^{57,58}$ The vast majority of these affect codon 918, although they have also been identified in few other regions of the gene. Some of these somatic mutations have heterogeneous distribution within the tumor or are detected only in a subset of metastatic nodules, raising concerns that they may not be essential for carcinogenesis. $^{58}$

\section{Targeted therapies}

Well-differentiated papillary and follicular carcinomas typically have indolent behavior and can be effectively treated by surgery followed by radioiodine therapy. However, tumors that lose differentiation and therefore the ability to trap radioiodine or unresectable follicular cell-derived tumors together with C-cell-derived medullary carcinomas do not respond to radioiodine treatment and usually have a much less favorable prognosis. These tumors are obvious candidates for alternative therapeutic approaches such as molecularly targeted therapy.

Several small-molecule tyrosine kinase inhibitors directed toward RET kinase have been tested in preclinical and clinical studies. ZD6474, an orally active low-molecular-weight receptor tyrosine kinase inhibitor, is a potent inhibitor of the vascular endothelial growth factor receptor 2 (VEGFR-2) and effectively blocks RET tyrosine kinase. ${ }^{59}$ ZD6474 has been shown to block phosphorylation and signaling from RET/PTC3 and RET carrying the most common
MEN2A and MEN2B mutations in vitro, to induce growth arrest of human papillary carcinoma cell lines carrying RET/PTC1 and to prevent tumor growth in nude mice after injection of RET/PTC3transformed fibroblasts or RET mutation-positive medullary carcinoma cells. ${ }^{60,61}$

Some evidence of response to ZD6474 therapy has been reported in patients with metastatic familial medullary carcinoma in clinical trials. ${ }^{59} \mathrm{~A}$ multicenter phase II double-blinded study is currently open to compare the efficacy of ZD6474 (ZACTIMA $^{\text {TM }}$ AstraZeneca Pharmaceuticals, DE, USA) vs placebo in patients with unresectable locally advanced or metastatic medullary carcinoma. This large and well-controlled study is expected to provide conclusive evidence for the therapeutic efficacy of this compound in inherited and sporadic medullary thyroid carcinomas.

Since antitumor activity of ZD6474 is likely due to a combination of its anti-RET activity and antiangiogenic activity mediated by blocking VEGFR, it will be of importance to find whether the extent of the therapeutic response to ZD6474 depends on the presence of RET mutation and its type. In preclinical studies, ZD6474 has been shown to inhibit most of the mutated variants of RET, except for the V804L and V804M mutations. ${ }^{62}$ V804 in the RET protein corresponds to the gate-keeper residues of ABL, PDGFR, c-KIT, and EGFR kinases, and mutations at these residues are known to confer resistance to various inhibitors. ${ }^{63}$ These results suggest that RET V804L and V804M mutations in medullary carcinomas may mediate primary resistance to ZD6474. ${ }^{62}$

Two other small-molecule tyrosine kinase inhibitors, the pyrazolopyrimidine compounds PP1 and PP2, have been tested in preclinical studies and found to be effective in therapeutic concentrations in blocking RET/PTC signaling in vivo and abolishing its tumorigenic effects in experimental animals. ${ }^{64,65}$ A multikinase inhibitor SU12248 (Sunitinib) has been shown to effectively inhibit signaling from RET/PTC kinase in the experimental models and has been progressed to phase II clinical trial in radioiodine-refractory, unresectable differentiated thyroid cancer and medullary thyroid cancer. $^{66}$

Various BRAF inhibitors have been identified and tested as potential therapeutic agents. They would be particularly valuable for thyroid cancer treatment due to the high frequency of $B R A F$ mutation in these tumors and its association with tumor dedifferentiation and resistance to the conventional radioiodine therapy. Moreover, since in the signaling cascade, BRAF is downstream of RET and RAS, BRAF inhibitors may be potentially effective in tumors with other mutations affecting this signaling pathway

BAY 43-9006 is a multikinase inhibitor with potent activity against RAF, VEGFR-2, VEGFR-3, PDGFR $\beta$, FLT-3, and c-KIT kinases. ${ }^{67}$ Orally administered BAY 43-9006 has shown strong antitumor 
effect in xenograft models of several types of cancer, and this effect is believed to be due to inhibition of MAPK signaling by blocking RAF and of angiogenesis by blocking VEGFR-2, VEGFR-3, and other kinases involved in tumor neovascularization. ${ }^{67}$ Importantly for the thyroid cancer field, BAY 43-9006 effectively blocks the wild-type BRAF and the mutant V600E BRAF kinase activity, although its effect on V600E BRAF is slightly less potent than on the wild-type BRAF kinase. ${ }^{8,67}$ BAY 43-9006 inhibits the BRAF signaling and growth of all thyroid cell lines carrying the mutant $B R A F^{68}$ It impaired the growth of the anaplastic carcinoma cell line xenografts in nude mice, and large areas of necrosis were found in the xenografts after the treatment of animals for 5 days. ${ }^{68}$ The inhibition of growth was mainly a cytostatic effect due to the cell arrest in G1 phase, and more profound cell death could be mediated by the inhibition of other kinases, especially those involved in angiogenesis. More recently, the therapeutic effect of BAY 43-9006 has also been found on cells carrying the activated forms of RET, including RET/PTC. ${ }^{69}$

BAY 43-9006 has been tested in clinical trials for several cancer types, including thyroid cancer. Preliminary results of the trial in patients with progressive papillary carcinoma have shown minimal or partial response in some patients. ${ }^{70,71}$ However, the complete results of this study as well other ongoing phase II trials of BAY 43-9006 (Sorafenib) in anaplastic thyroid carcinoma and metastatic medullary thyroid carcinoma are yet to be released. It will be important to find whether the response to BAY 43-9006 correlates with tumor phenotype and presence of $B R A F$ or other mutations.

The effects of other inhibitors of RAF kinases, AAL-881 and LBT-613, have been explored in thyroid cells in preclinical studies. ${ }^{72}$ Both compounds were found to block MAPK signaling and growth of rat thyroid cells and human thyroid tumor cell lines harboring the V600E BRAF and RET/PTC1. Suppression of the growth of BRAF mutant tumor xenografts in nude mice was also noted. However, some of these anticancer effects may be due to off-target effects, since they also occurred in the absence of inhibition of MEK and ERK phosphorylation. $^{72,73}$

Additional and potentially very effective therapeutic targets along the MAPK pathway are located downstream of BRAF. A non-ATP competitive MEK inhibitor CI-1040 has been found to abrogate tumor growth in BRAF mutant xenografts derived from various tumor types and has progressed to clinical trials. ${ }^{74}$

As the results of ongoing clinical trials are expected to be available in the near future and testing of novel kinase inhibitors continues, this information is expected to allow more precise assessment of the role of molecular inhibitors, administered alone or in combination, in the therapy of thyroid cancer.

\section{Acknowledgement}

This study was supported by NIH Grant R01 CA88041.

\section{References}

1 Hundahl SA, Fleming ID, Fremgen AM, et al. A national cancer data base report on 53856 cases of thyroid carcinoma treated in the US, 1985-1995. Cancer 1998;83:2638-2648.

2 Kimura ET, Nikiforova MN, Zhu Z, et al. High prevalence of BRAF mutations in thyroid cancer: genetic evidence for constitutive activation of the RET/PTC-RAS-BRAF signaling pathway in papillary thyroid carcinoma. Cancer Res 2003;63:1454-1457.

3 Fagin JA. Genetics of papillary thyroid cancer initiation: implications for therapy. Trans Am Clin Climatol Assoc 2005;116:259-269; discussion 269-271.

4 Kondo T, Ezzat S, Asa SL. Pathogenetic mechanisms in thyroid follicular-cell neoplasia. Nat Rev 2006;6: 292-306.

5 Cohen Y, Xing M, Mambo E, et al. BRAF mutation in papillary thyroid carcinoma. J Natl Cancer Inst 2003;95:625-627.

6 Xing M. BRAF mutation in thyroid cancer. Endocr Relat Cancer 2005;12:245-262.

7 Ciampi R, Nikiforov YE. Alterations of the BRAF gene in thyroid tumors. Endocr Pathol 2005;16:163-172.

8 Wan PT, Garnett MJ, Roe SM, et al. Mechanism of activation of the RAF-ERK signaling pathway by oncogenic mutations of B-RAF. Cell 2004;116:855-867.

9 Adeniran AJ, Zhu Z, Gandhi $\mathrm{M}$, et al. Correlation between genetic alterations and microscopic features, clinical manifestations, and prognostic characteristics of thyroid papillary carcinomas. Am J Surg Pathol 2006;30:216-222.

10 Nikiforova MN, Kimura ET, Gandhi M, et al. BRAF mutations in thyroid tumors are restricted to papillary carcinomas and anaplastic or poorly differentiated carcinomas arising from papillary carcinomas. J Clin Endocrinol Metab 2003;88:5399-5404.

11 Namba H, Nakashima M, Hayashi T, et al. Clinical implication of hot spot BRAF mutation, V599E, in papillary thyroid cancers. J Clin Endocrinol Metab 2003;88:4393-4397.

12 Xing M, Westra WH, Tufano RP, et al. BRAF mutation predicts a poorer clinical prognosis for papillary thyroid cancer. J Clin Endocrinol Metab 2005;90: 6373-6379.

13 Kim TY, Kim WB, Rhee YS, et al. The BRAF mutation is useful for prediction of clinical recurrence in lowrisk patients with conventional papillary thyroid carcinoma. Clin Endocrinol (Oxf) 2006;65:364-368.

14 Riesco-Eizaguirre G, Gutierrez-Martinez P, GarciaCabezas MA, et al. The oncogene BRAF V600E is associated with a high risk of recurrence and less differentiated papillary thyroid carcinoma due to the impairment of $\mathrm{Na}^{+} / \mathrm{I}^{-}$targeting to the membrane. Endocr Relat Cancer 2006;13:257-269.

15 Trovisco V, Vieira de Castro I, Soares P, et al. BRAF mutations are associated with some histological types of papillary thyroid carcinoma. J Pathol 2004;202: 247-251. 
16 Carta C, Moretti S, Passeri L, et al. Genotyping of an Italian papillary thyroid carcinoma cohort revealed high prevalence of BRAF mutations, absence of RAS mutations and allowed the detection of a new mutation of BRAF oncoprotein (BRAF(V599lns)). Clin Endocrinol (Oxf) 2006;64:105-109.

17 Hou P, Liu D, Xing M. Functional characterization of the T1799-1801del and A1799-1816ins BRAF mutations in papillary thyroid cancer. Cell Cycle 2007;6: 377-379.

18 Ciampi R, Knauf JA, Kerler R, et al. Oncogenic AKAP9BRAF fusion is a novel mechanism of MAPK pathway activation in thyroid cancer. J Clin Invest 2005;115: 94-101.

19 Begum S, Rosenbaum E, Henrique R, et al. BRAF mutations in anaplastic thyroid carcinoma: implications for tumor origin, diagnosis and treatment. Mod Pathol 2004;17:1359-1363.

20 Fusco A, Grieco M, Santoro M, et al. A new oncogene in human thyroid papillary carcinomas and their lymph-nodal metastases. Nature 1987;328:170-172.

21 Grieco M, Santoro M, Berlingieri MT, et al. PTC is a novel rearranged form of the ret proto-oncogene and is frequently detected in vivo in human thyroid papillary carcinomas. Cell 1990;60:557-563.

22 Nikiforov YE. RET/PTC rearrangement in thyroid tumors. Endocr Pathol 2002;13:3-16.

23 Tallini G, Asa SL. RET oncogene activation in papillary thyroid carcinoma. Adv Anat Pathol 2001;8:345-354.

24 Santoro M, Dathan NA, Berlingieri MT, et al. Molecular characterization of RET/PTC3; a novel rearranged version of the RET proto-oncogene in a human thyroid papillary carcinoma. Oncogene 1994;9:509-516.

25 Knauf JA, Kuroda H, Basu S, et al. RET/PTC-induced dedifferentiation of thyroid cells is mediated through Y1062 signaling through SHC-RAS-MAP kinase. Oncogene 2003;22:4406-4412.

26 Santoro M, Melillo RM, Grieco M, et al. The TRK and RET tyrosine kinase oncogenes cooperate with ras in the neoplastic transformation of a rat thyroid epithelial cell line. Cell Growth Differ 1993;4:77-84.

27 Jhiang SM, Sagartz JE, Tong Q, et al. Targeted expression of the ret/PTC1 oncogene induces papillary thyroid carcinomas. Endocrinology 1996;137:375-378.

28 Santoro M, Chiappetta G, Cerrato A, et al. Development of thyroid papillary carcinomas secondary to tissue-specific expression of the RET/PTC1 oncogene in transgenic mice. Oncogene 1996;12:1821-1826.

29 Powell Jr DJ, Russell J, Nibu K, et al. The RET/PTC3 oncogene: metastatic solid-type papillary carcinomas in murine thyroids. Cancer Res 1998;58:5523-5528.

30 Melillo RM, Castellone MD, Guarino V, et al. The RET/ PTC-RAS-BRAF linear signaling cascade mediates the motile and mitogenic phenotype of thyroid cancer cells. J Clin Invest 2005;115:1068-1081.

31 Mitsutake N, Knauf JA, Mitsutake S, et al. Conditional BRAFV600E expression induces DNA synthesis, apoptosis, dedifferentiation, and chromosomal instability in thyroid PCCL3 cells. Cancer Res 2005;65: 2465-2473.

32 Zhu Z, Ciampi R, Nikiforova MN, et al. Prevalence of RET/PTC rearrangements in thyroid papillary carcinomas: effects of the detection methods and genetic heterogeneity. J Clin Endocrinol Metab 2006;91: 3603-3610.

33 Unger K, Zitzelsberger H, Salvatore G, et al. Heterogeneity in the distribution of RET/PTC rearrangements within individual post-Chernobyl papillary thyroid carcinomas. J Clin Endocrinol Metab 2004;89:4272-4279.

34 Nikiforov YE, Rowland JM, Bove KE, et al. Distinct pattern of ret oncogene rearrangements in morphological variants of radiation-induced and sporadic thyroid papillary carcinomas in children. Cancer Res 1997;57:1690-1694.

35 Namba H, Rubin SA, Fagin JA. Point mutations of ras oncogenes are an early event in thyroid tumorigenesis. Mol Endocrinol 1990;4:1474-1479.

36 Ezzat S, Zheng L, Kolenda J, et al. Prevalence of activating ras mutations in morphologically characterized thyroid nodules. Thyroid 1996;6:409-416.

37 Zhu Z, Gandhi M, Nikiforova MN, et al. Molecular profile and clinical-pathologic features of the follicular variant of papillary thyroid carcinoma. An unusually high prevalence of ras mutations. Am J Clin Pathol 2003;120:71-77.

38 Hara H, Fulton N, Yashiro T, et al. N-ras mutation: an independent prognostic factor for aggressiveness of papillary thyroid carcinoma. Surgery 1994;116: 1010-1016.

39 Lemoine NR, Mayall ES, Wyllie FS, et al. High frequency of ras oncogene activation in all stages of human thyroid tumorigenesis. Oncogene 1989;4: 159-164.

40 Suarez HG, du Villard JA, Severino M, et al. Presence of mutations in all three ras genes in human thyroid tumors. Oncogene 1990;5:565-570.

41 Motoi N, Sakamoto A, Yamochi T, et al. Role of ras mutation in the progression of thyroid carcinoma of follicular epithelial origin. Pathol Res Pract 2000;196: $1-7$.

42 Basolo F, Pisaturo F, Pollina LE, et al. N-ras mutation in poorly differentiated thyroid carcinomas: correlation with bone metastases and inverse correlation to thyroglobulin expression. Thyroid 2000;10:19-23.

43 Garcia-Rostan G, Zhao H, Camp RL, et al. ras mutations are associated with aggressive tumor phenotypes and poor prognosis in thyroid cancer. J Clin Oncol 2003;21:3226-3235.

44 Fagin JA. Minireview: branded from the start-distinct oncogenic initiating events may determine tumor fate in the thyroid. Mol Endocrinol 2002;16:903-911.

45 Saavedra HI, Knauf JA, Shirokawa JM, et al. The RAS oncogene induces genomic instability in thyroid PCCL3 cells via the MAPK pathway. Oncogene 2000;19:3948-3954.

46 Kroll TG, Sarraf P, Pecciarini L, et al. PAX8-PPARgamma1 fusion oncogene in human thyroid carcinoma [corrected]. Science 2000;289:1357-1360.

47 French CA, Alexander EK, Cibas ES, et al. Genetic and biological subgroups of low-stage follicular thyroid cancer. Am J Pathol 2003;162:1053-1060.

48 Nikiforova MN, Lynch RA, Biddinger PW, et al. RAS point mutations and PAX8-PPAR gamma rearrangement in thyroid tumors: evidence for distinct molecular pathways in thyroid follicular carcinoma. J Clin Endocrinol Metab 2003;88:2318-2326.

49 Dwight T, Thoppe SR, Foukakis T, et al. Involvement of the PAX8/peroxisome proliferator-activated receptor gamma rearrangement in follicular thyroid tumors. J Clin Endocrinol Metab 2003;88:4440-4445.

50 Nikiforova MN, Biddinger PW, Caudill CM, et al. PAX8-PPARgamma rearrangement in thyroid tumors: RT-PCR and immunohistochemical analyses. Am J Surg Pathol 2002;26:1016-1023. 
51 Gregory Powell J, Wang X, Allard BL, et al. The PAX8/ PPARgamma fusion oncoprotein transforms immortalized human thyrocytes through a mechanism probably involving wild-type PPARgamma inhibition. Oncogene 2004;23:3634-3641.

52 Giordano TJ, Au AY, Kuick R, et al. Delineation, functional validation, and bioinformatic evaluation of gene expression in thyroid follicular carcinomas with the PAX8-PPARG translocation. Clin Cancer Res 2006;12:1983-1993.

53 Reddi HV, McIver B, Grebe SK, et al. The paired box-8/ peroxisome proliferator-activated receptor-gamma oncogene in thyroid tumorigenesis. Endocrinology 2007;148:932-935.

54 Mulligan LM, Marsh DJ, Robinson BG, et al. Genotypephenotype correlation in multiple endocrine neoplasia type 2: report of the International RET Mutation Consortium. J Intern Med 1995;238:343-346.

55 Hansford JR, Mulligan LM. Multiple endocrine neoplasia type 2 and RET: from neoplasia to neurogenesis. J Med Genet 2000;37:817-827.

56 Santoro M, Melillo RM, Carlomagno F, et al. Molecular biology of the MEN2 gene. J Internal Med 1998;243: 505-508.

57 Alberti L, Carniti C, Miranda C, et al. RET and NTRK1 proto-oncogenes in human diseases. J Cell Physiol 2003;195:168-186.

58 Eng C, Mulligan LM, Healey CS, et al. Heterogeneous mutation of the RET proto-oncogene in subpopulations of medullary thyroid carcinoma. Cancer Res 1996;56: 2167-2170.

59 Herbst RS, Heymach JV, O’Reilly MS, et al. Vandetanib (ZD6474): an orally available receptor tyrosine kinase inhibitor that selectively targets pathways critical for tumor growth and angiogenesis. Expert Opin Investig Drugs 2007;16:239-249.

60 Carlomagno F, Vitagliano D, Guida T, et al. ZD6474, an orally available inhibitor of KDR tyrosine kinase activity, efficiently blocks oncogenic RET kinases. Cancer Res 2002;62:7284-7290.

61 Santoro M, Carlomagno F. Drug insight: small-molecule inhibitors of protein kinases in the treatment of thyroid cancer. Nat Clin Pract 2006;2:42-52.

62 Carlomagno F, Guida T, Anaganti S, et al. Disease associated mutations at valine 804 in the RET receptor tyrosine kinase confer resistance to selective kinase inhibitors. Oncogene 2004;23:6056-6063.

63 Pao W, Miller VA. Epidermal growth factor receptor mutations, small-molecule kinase inhibitors, and nonsmall-cell lung cancer: current knowledge and future directions. J Clin Oncol 2005;23:2556-2568.

64 Carlomagno F, Vitagliano D, Guida T, et al. The kinase inhibitor PP1 blocks tumorigenesis induced by RET oncogenes. Cancer Res 2002;62:1077-1082.

65 Carlomagno F, Vitagliano D, Guida T, et al. Efficient inhibition of RET/papillary thyroid carcinoma oncogenic kinases by 4-amino-5-(4-chloro-phenyl)-7(t-butyl)pyrazolo[3,4-d]pyrimidine (PP2). J Clin Endocrinol Metab 2003;88:1897-1902.

66 Kim DW, Jo YS, Jung HS, et al. An orally administered multitarget tyrosine kinase inhibitor, SU11248, is a novel potent inhibitor of thyroid oncogenic RET/ papillary thyroid cancer kinases. J Clin Endocrinol Metab 2006;91:4070-4076.

67 Wilhelm SM, Carter C, Tang L, et al. BAY 43-9006 exhibits broad spectrum oral antitumor activity and targets the RAF/MEK/ERK pathway and receptor tyrosine kinases involved in tumor progression and angiogenesis. Cancer Res 2004;64:7099-7109.

68 Salvatore G, De Falco V, Salerno P, et al. BRAF is a therapeutic target in aggressive thyroid carcinoma. Clin Cancer Res 2006;12:1623-1629.

69 Carlomagno F, Anaganti S, Guida T, et al. BAY 43-9006 inhibition of oncogenic RET mutants. J Natl Cancer Inst 2006;98:326-334.

70 Baudin E, Schlumberger M. New therapeutic approaches for metastatic thyroid carcinoma. Lancet Oncol 2007;8:148-156.

71 Espinosa AV, Porchia L, Ringel MD. Targeting BRAF in thyroid cancer. Br J Cancer 2007;96:16-20.

72 Ouyang B, Knauf JA, Smith EP, et al. Inhibitors of Raf kinase activity block growth of thyroid cancer cells with RET/PTC or BRAF mutations in vitro and in vivo. Clin Cancer Res 2006;12:1785-1793.

73 Chiloeches A, Marais R. Is BRAF the Achilles' Heel of thyroid cancer? Clin Cancer Res 2006;12: 1661-1664.

74 Solit DB, Garraway LA, Pratilas CA, et al. BRAF mutation predicts sensitivity to MEK inhibition. Nature 2006;439:358-362. 